\title{
Births and perinatal deaths in Australia: variations by day of week
}

\author{
C D MATHERS \\ From the National Perinatal Statistics Unit, University of Sydney, Sydney, New South Wales, Australia
}

SUMmARY Analysis of births occurring in Australia during 1976-9 showed that they followed a seven-day cycle, being concentrated on Tuesdays to Fridays and least numerous on Sundays, which had $26 \%$ fewer births than expected. Stillbirth and neonatal death rates were $17 \%$ and $29 \%$ higher respectively among babies born at weekends than among those born on weekdays. The incidence of low birth weight was also significantly higher in the weekend group in New South Wales. The patterns seen in births and perinatal deaths are largely associated with the selective effects of intervention in delivery rather than with any variation in quality of care throughout the week.

Variations in the number of births according to the day of the week have been reported for births in Sydney ${ }^{1}$ and Canberra. ${ }^{2}$ Similar variations have been reported in the United States ${ }^{3}$ and England and Wales. ${ }^{4}$ The latter paper also notes variations in perinatal mortality rates according to the day of the week.

This paper examines the distribution of births and perinatal deaths by day of week for all births occurring in Australia during the four years 1976 to 1979. Some implications of the observed patterns of births and deaths are discussed.

\section{Methods}

Computer tapes containing data relating to births and perinatal deaths registered in the years $1976-80$ in Australia were supplied by the Australian Bureau of Statistics. All subsequent analyses refer to the cohort of infants born in the years 1976-9 and registered by the end of 1980 (898 596 live births and 7657 stillbirths). As most published tables use births and deaths registered in given years, the numbers and rates presented here will differ from published data. Definitions used here are the same as those used in publications ${ }^{5}$ of the Australian Bureau of Statistics. Perinatal deaths comprise fetal deaths (stillbirths) and neonatal deaths (deaths within 28 days of birth). This differs from the definition of the perinatal period used in the United Kingdom and the United States, where it extends only to the seventh day after birth. The definition of perinatal death was amended in 1979 to include only infants and fetuses weighing at least $500 \mathrm{~g}$ or of at least 22 weeks' gestational age if birth weight is unavailable. Tabulations presented below exclude perinatal deaths before 1979 that do not satisfy these criteria. Under the new definition, there were 15013 perinatal deaths in the 1976-9 birth cohort.

Counts were made of the numbers of births occurring on gazetted public holidays. These include Christmas Day and Boxing Day (where not gazetted) and Easter Friday, Saturday, and Monday. In calculating expected numbers of births on particular public holidays such as Christmas the seasonal distribution of births throughout the year was also taken into account.

Neonatal death rates were calculated for day of birth cohorts and also for day of death cohorts. Date of birth is not transferred from the perinatal death certificate to computer by the Australian Bureau of Statistics, so it is not possible to tabulate directly neonatal deaths by day of birth. Nevertheless, age at death is coded to within minutes under one hour, hours under one day, and days under one month. Date of birth is calculated by subtracting age at death in completed days from date of death. If births are uniformly distributed throughout the day the calculated date of birth will be in error by one day for $9 \%$ of deaths occurring under one day of age, for $40 \%$ of deaths occurring from one to six completed days, and for $50 \%$ of deaths over one week.

The calculated date of birth was used to compute age-stratified "apparent" neonatal death rates for day of birth cohorts. Using the age-dependent misclassification rate, it is then possible to estimate the true death rate distribution for each age and hence the underlying neonatal death rates for each 
day of week birth cohort. These underlying rates are not altered appreciably if the distribution of births through the day is assumed to be non-uniform, resulting in slight changes in the misclassification rates.

To calculate the neonatal death rates by day of death, it was necessary to calculate the population at risk of dying on each day of the week. As will be shown below, the risk of death varies significantly according to day of birth, and as the great majority of neonatal deaths occur within a day or two of birth, the dependence of the neonatal death rate on day of week of death will be masked by its dependence on day of week of birth. This confounding effect of day of birth was removed by calculating the expected number of deaths on each day of the week using the distribution of births by day of the week, the observed total distribution of deaths by age at death, and the observed modification of the risk of death according to the day of birth.

Differences between observed and expected numbers of births and deaths were tested for statistical significance using the normal approximation to the binomial distribution to test the difference of two proportions. Significance levels given in this paper refer to the probability, $p$, that the observed difference is due to chance alone.

\section{Results}

\section{BIRTHS}

Figure 1 shows the distribution by day of week for all live births occurring in Australia in the years 1976-9. The percentage deviation of the observed births a day from the average of 615 a day for all days of the week shows a pronounced pattern of excess births on Tuesday to Friday peaking on Thursday and a $21 \%$ deficit of births at the weekend, this deficit being even higher (26\%) on Sundays. There are $52 \%$ more births on Thursdays than on Sundays.

The average number of births on public holidays is $15 \%$ fewer than the average for all days. Nearly $60 \%$ of gazetted public holidays fall on a Monday, and on these days there are $18 \%$ fewer births than on ordinary Mondays. If public holidays are excluded

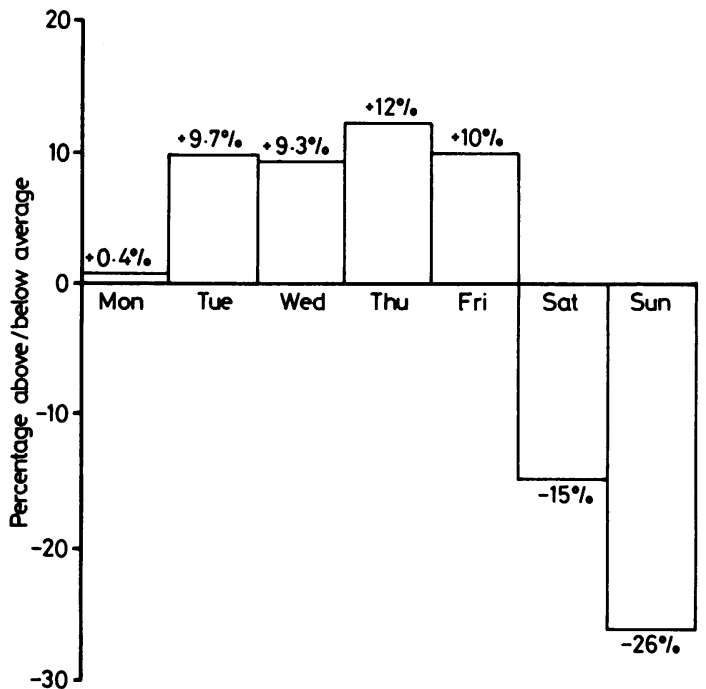

Fig 1 Distribution by day of week of all live births in Australia in 1976-9. Percentage deviation of observed number of births a day from average for all days is plotted.

the day of week distribution shown in fig 1 changes appreciably only for Mondays, where the excess rises from $0 \cdot 4 \%$ to $2 \cdot 5 \%$.

There were 29\% fewer births on Christmas Days in 1976-9 than expected on the corresponding days ofo the week and no apparent excess of births on the? three days preceding Christmas. There was an average of $4 \%$ fewer births on Easter Friday to Monday (inclusive). This deficit was balanced by a 9\% excess on the Wednesday and Thursday preceding Easter.

Table 1 gives the distributions of observed births on all public holidays and on weekends excluding public holidays for each of the States and Territories. The weekend deficit ranges from a maximum of $24 \%$ in New South Wales to $11 \%$ in the Australian Capital Territory. The overall weekly pattern of births (excluding public holidays) is similar in New South Wales, Victoria, Queensland, and Western Australia. In South Australia, births reach a maximum on Friday $(+14 \%)$ rather than Thursdays.

Table 1 Average number of livebirths a day and deviations from the daily average for live births in Australia 1976-9

\begin{tabular}{|c|c|c|c|c|c|c|c|c|c|}
\hline & \multicolumn{9}{|c|}{ State or Territory } \\
\hline & $N S W$ & VIC & $Q L D$ & $S A$ & $W A$ & $T A S$ & $N T$ & $A C T$ & $A U S$ \\
\hline $\begin{array}{l}\text { Average daily births } \\
\text { Deviations below average (\%) }\end{array}$ & 211 & 161 & 96 & 51 & 57 & $18 \cdot 7$ & $7 \cdot 1$ & $11 \cdot 6$ & 615 \\
\hline $\begin{array}{l}\text { Weekend* } \\
\text { Sunday* } \\
\text { Public holidays }\end{array}$ & $\begin{array}{l}24 \\
28 \\
21\end{array}$ & $\begin{array}{l}18 \\
25 \\
13\end{array}$ & $\begin{array}{l}22 \\
29 \\
20\end{array}$ & $\begin{array}{l}20 \\
26 \\
15\end{array}$ & $\begin{array}{r}20 \\
27 \\
7\end{array}$ & $\begin{array}{l}15 \\
22 \\
18\end{array}$ & $\begin{array}{l}14 \\
20 \\
13\end{array}$ & $\begin{array}{r}11 \\
17 \\
7\end{array}$ & $\begin{array}{l}20 \\
26 \\
15\end{array}$ \\
\hline
\end{tabular}

Excluding public holidays.

NSW = New South Wales; VIC = Victoria; QLD = Queensland; SA = South Australia; WA = Western Australia; TAS = Tasmania; NT = Northern Territory; ACT = Australian Capital Territory; AUS = Australia. 


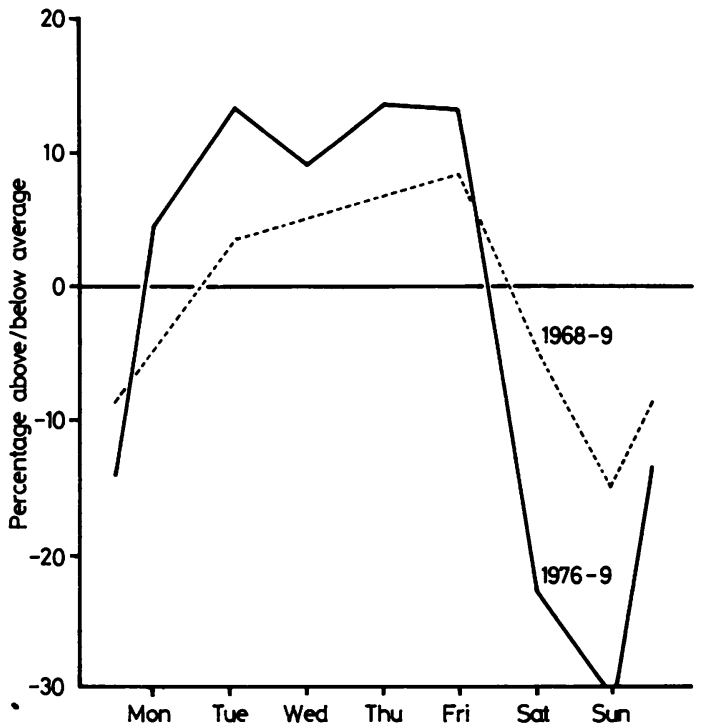

Fig 2 Distribution by day of week of live births to mothers resident in the Sydney Metropolitan region in 1976-9 and 1968-9 (from Martins). ${ }^{1}$

There are no excess births on Wednesdays $(+0 \cdot 7 \%)$ in Tasmania, on Thursdays $(-0.7 \%)$ in the Northern Territory and on Mondays $(+0.7 \%)$ and Tuesdays (0\%) in the Australian Capital Territory. The pattern of metropolitan and other births were compared. Differences were slight with an average of $3 \%$ fewer weekend births to metropolitan mothers than to non-metropolitan mothers.

The weekly pattern of births in 1976-9 to mothers resident in Sydney is compared in fig 2 with that found by Martins ${ }^{1}$ for births in 1968 and 1969 . The Sunday deficit has doubled over this $\mathbf{1 0}$-year period from $15 \%$ to $30 \%$. The weekday peak has shifted

Table 2 Birthweight distribution by day of week of birth for live births in New South Wales 1976-9

\begin{tabular}{lllll}
\hline & Weekdays & Weekend & Difference & Significance \\
\hline Percentage not given & 5.9 & 5.6 & $-5 \%$ & $\mathrm{p}=0.001$ \\
Mean birthweight* & 3363 & 3339 & $-1 \%$ & $\mathrm{p}<0.001$ \\
Percentage $<2500 \mathrm{~g}^{*}$ & & & & \\
$\quad$ Total & 5.1 & 6.0 & $+18 \%$ & $\mathrm{p}<0.001$ \\
Sydney & 5.4 & 6.6 & $+22 \%$ & $\mathrm{p}<0.001$ \\
Other & 4.7 & 5.3 & $+13 \%$ & $\mathrm{p}<0.001$ \\
\hline
\end{tabular}

Excluding births where birth weight not given. from Friday to Thursday. No consistent time trends are apparent within the four-year period 1976-9.

Information on birth weight in live births has been collected in New South Wales from 1975 onwards, with a completeness of $94 \%$ for births occurring in 1976-9. Table 2 summarises the distributions of mean birth weight and low birth weight $(<2500 \mathrm{~g})$ by day of week. The incidence of low birth weight is $5.1 \%$ in weekday births and $6 \%$ in weekend births. The difference between these rates is highly significant and is larger for births to mothers resident in Sydney than for other births in New South Wales.

\section{PERINATAL DEATHS}

Table 3 summarises the distribution of stillbirths by day of week for 1976-9. The stillbirth rates on the weekdays Monday to Friday varies little, with an average rate of 8.2 per 1000 total births. This rate is higher on Saturdays $(10 \cdot 1 / 1000)$ and Sundays $(9 \cdot 0 / 1000)$. The difference between the weekend and weekday rates is highly significant. In metropolitan areas the stillbirth rate is high on Saturdays but essentially the same on all other days. This contrasts with non-metropolitan areas, where the Saturday and Sunday rates are similar and 25\% higher than the weekday rate.

Table 4 compares the neonatal death rates for babies born on weekdays and at the weekend. The overall neonatal death rate for babies born at the weekend is $29 \%$ higher than that for babies born on weekdays. This difference is even greater (35\%) for births to mothers resident in metropolitan areas. Table 4 also analyses neonatal death rates according to whether the main certified condition in the infant was a congenital malformation or not. Estimated rates are also given for neonatal deaths in low birthweight infants and in other infants. The denominators for these rates have been estimated by assuming that the birthweight distribution by day of week for all Australian livebirths is the same as that for births in New South Wales (table 2). Table 5 gives a further analysis of neonatal deaths due to causes other than malformations in low birthweight infants. All these rates are significantly higher $(p<0.001)$ for infants born at weekends.

Table 6 compares observed neonatal deaths a day on weekdays and at the weekend with the average for all days. To determine whether day of the week of death influences the death rate it is necessary to

Table 3 Stillbirth rate (per 1000 total births) by day of week for Australia 1976-9

\begin{tabular}{|c|c|c|c|c|c|}
\hline & Weekdays & Saturday & Sunday & Weekend/weekday difference & Significance \\
\hline $\begin{array}{l}\text { Metropolitan } \\
\text { Other }\end{array}$ & $\begin{array}{l}8 \cdot 7 \\
7 \cdot 5\end{array}$ & $\begin{array}{r}10 \cdot 7 \\
9.4\end{array}$ & $\begin{array}{l}8 \cdot 5 \\
9 \cdot 3\end{array}$ & $\begin{array}{l}+10 \% \\
+25 \%\end{array}$ & $\begin{array}{l}p=0.002 \\
p<0.001\end{array}$ \\
\hline Total & $8 \cdot 2$ & $10 \cdot 1$ & $9 \cdot 0$ & $+17 \%$ & $p<0.001$ \\
\hline
\end{tabular}


Table 4 Neonatal death rates (per 1000 live births) by day of week of birth for Australia 1976-9

\begin{tabular}{|c|c|c|c|c|}
\hline & Weekdays & Weekend & Difference & Significance \\
\hline $\begin{array}{l}\text { Deaths due to } \\
\text { malformations }\end{array}$ & $2 \cdot 47$ & $2 \cdot 57$ & $+4 \%$ & NS \\
\hline $\begin{array}{l}\text { Deaths due to other } \\
\text { causes }\end{array}$ & $5 \cdot 20$ & $7 \cdot 30$ & $+40 \%$ & $p<0.001$ \\
\hline Birth weight $<2500 \mathrm{~g}^{*}$ & 89.7 & $\begin{array}{r}105 \cdot 9 \\
3.25\end{array}$ & $+18 \%$ & $p<0.001$ \\
\hline Early neonatal & $\begin{array}{l}6.52 \\
1.15\end{array}$ & $8 \cdot 40$ & $+29 \%$ & $\mathrm{p}<0.001$ \\
\hline \multicolumn{5}{|l|}{ Total } \\
\hline neonatal deaths & $7 \cdot 67$ & $9 \cdot 87$ & $+29 \%$ & $\mathrm{p}<0.001$ \\
\hline
\end{tabular}

*Denominator estimated assuming the birthweight distribution in live births is the same as that for New South Wales.

Table 5 Neonatal death rates* (per 1000 live births) by day of week of birth for deaths due to causes other than congenital malformations in low birthweight infants

\begin{tabular}{llll}
\hline & Weekdays & Weekend & Difference \\
\hline Early neonatal & 47.5 & $55 \cdot 7$ & $+17 \%$ \\
Late neonatal & $22 \cdot 8$ & 32.8 & $+44 \%$ \\
Total neonatal & 70.3 & 88.5 & $+26 \%$ \\
\hline
\end{tabular}

"Denominators estimated using New South Wales birthweight distribution.

Table 6 Neonatal deaths per day by day of week of death for all infants born in 1976-9

\begin{tabular}{llll}
\hline Age at death & Weekdays & Weekend & All days \\
\hline$<1$ day & 2.90 & 2.70 & 2.84 \\
1-6 days & 1.45 & 1.43 & 1.44 \\
$7-27$ days & 0.74 & 0.75 & 0.75 \\
$<28$ days & 5.09 & 4.89 & 5.03 \\
\hline
\end{tabular}

account correctly for the influence of day of birth. The distribution of births by day of week affects the observed deaths by day of week in two ways. Firstly, since there are fewer births at weekends and most deaths occur within a day or two of birth, there will be fewer early neonatal deaths at weekends than otherwise expected. Secondly, infants born at the weekend have a significantly higher neonatal death rate than others. When both these effects are taken into account, as described in the methods section, the neonatal death rate was found to vary by less than $2 \%$ throughout the week. This variation is not statistically significant.

\section{Discussion}

An analysis of live births in Sydney in 1968-9 by Martins ${ }^{1}$ showed that the numbers of births occurring on Sundays and public holidays were $15 \%$ and $18 \%$ fewer respectively than the overall daily average. Ten years later, the Sunday deficit has risen to $30 \%$ in Sydney and $26 \%$ nationally. The deficit of births on public holidays has risen to $22 \%$ in Sydney.
Macfarlane $e^{4}$ has documented a similar fall in the relative frequency of weekend births in England and Wales, where the Sunday deficit rose from $8 \%$ in $1963-7$ to $12 \%$ in 1970 and $23 \%$ in 1976 .

Such increases in the weekend deficit of births over one decade make it difficult to argue that the weekly birth pattern is due to a biological cycle, either in the timing of the onset of spontaneous labour or in probability of conception. Such explanations are rendered even more untenable by the observed deficits of births on public holidays in Australia.

A more likely explanation for these patterns is the increasing prevalence of obstetric intervention in the timing of delivery, either through induction of labour or elective caesarean section. There has been a pronounced increase in the rate of obstetric intervention since the early 'sixties in England and Wales. ${ }^{4}$ A similar increase appears to have occurred in Australia. The intervention rate for private patients in a Sydney teaching hospital ${ }^{6}$ rose from $14 \%$ in $1963-5$ to $37 \%$ in $1973-5$. For the insured population of South Australia, ${ }^{7}$ it rose from $9 \%$ in 1963 to $42 \%$ in 1978. The induction rate at a Victorian teaching hospital rose from $6 \%$ in 1968 to $15 \%$ in 1976 for booked-that is, non-emergency-public patients. ${ }^{8}$

Intervention rates vary with State, insurance statu\& of patient, size of hospital, and type of medicas practice. In a Brisbane teaching hospital in 1972the overall frequencies of induction and caesaria section were $29 \%$ and $11 \%$ respectively in public patients and $34 \%$ and $23 \%$ respectively in private patients. ${ }^{9}$ From a sample of medical benefits claims covering roughly one-third of births in Victoria in $1977-8$, the induction rate was $21 \%$ and the caesarian section rate $12 \% .^{8}$ Tasmania is the only State that as yet publishes comprehensive data on obstetric procedures for all births. ${ }^{10}$ The induction rate in Tasmania was $32 \%$ for $1976-80$ with a peak rate of $37 \%$ in 1977 declining to $26 \%$ in 1980 . The overall incidence of caesarian section rose from $6 \%$ in 1976 to $8 \%$ in 1980 .

There is some evidence that the obstetric intervention rate is lower in small hospitals, ${ }^{10}$ suggesting that the Sunday deficit may be smaller for births in country areas than in metropolitan areas. Indeed, the Sunday deficit of births for mothers resident in rural local government areas where the population of the largest town did not exceed 10000 was $23 \%$ compared with $26 \%$ for mothers resident in metropolitan areas in Australia. The deficits for New South Wales were somewhat larger at $24 \%$ for rural residents and 30\% for Sydney residents.

The unusual pattern of births in Tasmania, the Northern Territory, and the Australian Capital 6 Territory is also more readily explained in terms of 
elective intervention than other explanations invoking biological cycles. One would expect the effect of weekly patterns in medical practice to be more visible in the smaller States with more centralised obstetric facilities.

Macfarlane ${ }^{4}$ also reported higher perinatal mortality rates among babies born at weekends in England and Wales. A study of mortality in infants of very low birth weight in South-east London also found a raised mortality at weekends. ${ }^{11}$

There are several possible explanations for the variations in perinatal mortality by day of week found in these studies and also in the Australian birth cohort analysed in this paper. These explanations are not necessarily mutually exclusive. One possible explanation is that quality of care is lower at weekends when staffing levels are lower and specialist obstetric services are less readily available. Another possibility, suggested by Macfarlane,${ }^{4}$ is that elective intervention may act to concentrate low-risk births at term on to weekdays. Although the probability of intervention is greater in high-risk pregnancies, intervention is acting in the larger numbers of low-risk pregnancies to shift a proportion of their deliveries from weekend to weekdays. Direct evidence supporting this hypothesis is provided by the increased incidence of low birth weight in weekend deliveries in New South Wales. Quality of care at and after birth will not influence the birth weight distribution, whereas selective use of induction will.

The neonatal death rate was $29 \%$ higher for babies born at the weekends than on weekdays. Since most neonatal deaths occur within a day or two of birth, this could be due to lower levels of medical care at the weekend. The late neonatal death rate, however, was raised by the same proportion as the early neonatal death rate for babies born at weekends. For deaths due to causes other than malformations in low birthweight infants, the late neonatal death rate was raised by a greater factor than the early neonatal rate for babies born at weekends. Since late neonatal deaths occur at a slowly declining rate from seven to 27 days after birth, variations in quality of care by day of week is unlikely to give rise to any variation in the late neonatal death rate by date of birth.

Conversely, selective intervention practices would have a negligible effect on variations in the day of death distribution of late neonatal deaths. Such variations could be most easily explained in terms of quality of care. As shown in table 6, there is no difference between the proportion of late neonatal deaths occurring on weekdays and at weekends.

It would thus appear that variations in quality of care throughout the week have no demonstrable effects on the patterns of morbidity and neonatal mortality. The data presented above on births, low birth weight, and neonatal mortality all support, albeit in a circumstantial way, the hypothesis that elective intervention is a principal factor in producing the observed day of week patterns.

Lumley $^{8}$ has estimated that one-third of inductions in Tasmania in 1974-7 were carried out for accepted medical indications according to the stated complications of pregnancy. Obstetricians and general practitioners also use induction to ensure that labour and delivery of women with risk factors take place during daylight hours, when all obstetric services are readily available to deal with emergencies. Other factors contributing to excess births on weekdays include elective caesarian section, hospital staffing policies, and the convenience of medical practitioners and of women and their families. ${ }^{12}$

The evidence presented here suggests that the overall result of obstetric practices is to concentrate deliveries of low-risk pregnancies during weekdays. As shown in table 4, this selection effect operates preferentially on babies whose birth weight is not low, but not on babies with lethal congenital malformations.

The indirect nature of the data presented in this article highlights the need for population-based data collections that would allow direct analysis of the impact of obstetric practices on reproductive patterns and outcomes in Australia. Most Australian States have now introduced a perinatal morbidity form which, among other things, will provide such data on the incidence and impact of elective intervention in labour and delivery.

I thank the Australian Bureau of Statistics for the provision of data and my colleagues in the National Perinatal Statistics Unit for helpful discussions. The National Perinatal Statistics Unit is funded by a Health Services Research and Development grant from the Commonwealth Department of Health.

\section{References}

${ }^{1}$ Martins JM. Never on Sundays! Med J Aust 1972; 1: 487.

${ }^{2}$ Wells R. Still never on Sundays! Med J Aust 1980; 2: 160.

${ }^{3}$ Rindfuss RR, Ladinsky JL. Patterns of births: implications for the incidence of elective induction. Med Care 1976; 14: 685-93.

${ }^{4}$ Macfarlane A. Variations in number of births and perinatal mortality by day of week in England and Wales. Br Med J 1978; ii: 1670-3.

${ }^{5}$ Australian Bureau of Statistics. Perinatal deaths Australia 1980. Canberra: ABS, 1982.

- Jasper G. Active obstetrics for convenience. Med J Aust 1976; 2: 625 .

${ }^{7}$ Opit LJ, Selwood TS. Caesarean-section rates in Australia. Med J Aust 1979; 2: 706-9. 
${ }^{8}$ Lumley J. Patterns of obstetric intervention: Tasmania and Victoria. New Doctor 1980; 15: 27-9.

${ }^{\circ}$ Chang A, Keeping JD, Morrison J, Esler EJ. A comparison of obstetric morbidity and management between clinic and private patients. Aust NZ J Obstet Gynecol 1979; 19: 158-65.
${ }^{10}$ Carter LV, Dooley DJ, Elliott CE, Semple A. Obstetrics in small hospitals. Med J Aust 1980; 1: 359-62.

${ }^{11}$ Stanley FJ, Alberman ED. Infants of very low birthweight I. Perinatal factors affecting survival. Dev Med Child Neurol 1978; 20: 300-12.

${ }^{12}$ Anonymous. Induction of labour. $\mathrm{Br} \mathrm{Med} J$ 1976; i: 729-30. 